Artigo original

\title{
Vigilância em saúde do trabalhador: um estudo sobre acidentes graves do trabalho
}

\author{
Workers' health surveillance: a study of major accidents at work
}

Karini da Rosa ${ }^{1}$, Susimar Souza Rosa ${ }^{1}$, Priscila de Almeida ${ }^{1}$, Dayane Diehl' ${ }^{1}$, Suzane Beatriz

Frantz Krug1.

${ }^{1}$ Universidade de Santa Cruz do Sul, Santa Cruz do Sul, RS, Brasil.

\section{RESUMO}

Submissão: 01/09/2016

Aceite: $20 / 09 / 2016$

Justificativa e objetivo: Os acidentes do trabalho constituem o maior agravo à saúde dos trabalhadores brasileiros, assim, o objetivo do estudo é caracterizar dados sociodemográficos e ocupacionais de trabalhadores acometidos por acidentes graves do trabalho no municipio de Santa Cruz do Sul/RS. Método: trata-se de um estudo de campo, documental, retrospectivo, com coleta de dados em formulários de notificação epidemiológica da Unidade Municipal de Referência em Saúde do Trabalhador (UMREST), no período de 2008 a 2010. Resultados: Realizou-se levantamento de dados em 1.263 formulários de notificações de pacientes da UMREST, sendo encontrados 986 casos de acidente graves do trabalho, sendo que 78,59\% dos casos com trabalhadores do sexo masculino na faixa etária de 20 a 29 anos. No ano de 2008 houve maior ocorrência de casos notificados nos sistemas de informação da área de Saúde do Trabalhador. As ocupações com maior ocorrência foram os alimentadores de linha de produção $(13,48 \%)$, auxiliar de processamento de fumo $(8,0 \%)$, trabalhadores nos serviços de manutenção e conservação de edifícios e logradouros $(7,0 \%)$. Dentre os tipos de acidentes, encontrou-se $66,63 \%$ de acidentes típicos e $31,23 \%$ de acidentes de trajeto. Identificou-se a região central do município como a de maior ocorrência dos casos com 47,2\%, seguido pelo bairro Distrito Industrial com $27,1 \%$ dos casos. Conclusão: verificou-se que os acidentes acometeram, com maior frequência, trabalhadores homens, jovens, de instituições de trabalho localizadas na região central do município. Desta forma, ressalta-se a necessidade de ações de prevenção e assistência que atendam o perfil dos trabalhadores estudados.

DESCRITORES: Acidentes de trabalho. Vigilância em saúde do trabalhador. Saúde do trabalhador.

\section{ABSTRACT}

Background and objectives: Work accidents are the biggest health problem of Brazilian workers, thus, the objective of the study is to characterize sociodemographic and occupational data affected by serious accidents workers work in the municipality of Santa Cruz do Sul/RS. 
Method: this is a field study, documental, retrospective, with data collection in epidemiological report forms of the Municipal Unit Worker Health Reference (UMREST) in the period of 2008 to 2010. Results: We conducted data collection in 1263 patient notification forms of the UMREST, and found 986 cases of serious work accident, and $78.59 \%$ of cases with male workers aged 20 to 29 years. In 2008 there were more cases reported in the information systems of Occupational Health. The occupations with the highest occurrence were the production line feeders $(13,48 \%)$, tobacco processing assistant $(8.0 \%)$, workers in the maintenance and conservation of buildings and public parks (7.0\%). Among the types of accidents, $66.63 \%$ were typical accidents and $31.23 \%$ of commuting accidents. It identified the central area of the city as the most frequent cases with $47.2 \%$, followed by the Industrial District neighborhood with $27.1 \%$ of cases. Final Thoughts: it was found that the accidents occurred more often on male workers, young, from labor institutions located in the central area of the city. Thus, we emphasize the need for prevention and care actions that meet the profile of the workers studied.

KEYWORDS: Accidents at work. Occupational health surveillance. Occupational health.

\section{INTRODUÇÃO}

O trabalho é um elemento central na vida das pessoas, possibilita satisfação de necessidades, atendimento de desejos, mas também pode gerar insatisfação e sofrimento permanentes, devido a cargas físicas ou mentais presentes. ${ }^{1}$ Pode, em algumas situações adversas, ser causador de acidentes e doenças que ocorrem no exercício da atividade laboral ou no percurso de casa para o trabalho, do trabalho para a casa. ${ }^{2}$

Dentre os tantos agravos relacionados ao trabalho, encontra-se o acidente grave do trabalho que é todo aquele que acarreta mutilação física ou funcional, cuja natureza implique em comprometimento extremamente sério, que pode ter consequências nefastas ou fatais. Acidentes do trabalho grave são frequentes e, por isso, são importantes as ações de proteção e prevenção com o intuito de reduzir tal número, diminuir o número de mortes decorrentes dos processos e do ambiente de trabalho, bem como orientar e disponibilizar melhores condições de assistência à saúde a esses trabalhadores. ${ }^{3}$

No Brasil, os acidentes de trabalho grave são a maioria dos casos de afastamentos do trabalho, o que aponta a precariedade das medidas de segurança nos ambientes do trabalho. ${ }^{4}$ Além disso, os acidentes afetam socioeconomicamente a realidade do país e dos municípios. São milhares de pessoas que anualmente afastam-se do trabalho e passam a receber benefício da Previdência Social enquanto estão no processo de reabilitação. Há estimativas de que até $4 \%$ do produto interno bruto de um país estejam sendo gastos com acidentes e doenças ocupacionais e os problemas decorrentes, tornando-os um importante problema de saúde pública a nível mundial, inclusive. ${ }^{5}$ 
Nessa realidade do mundo do trabalho, o tema Vigilância em Saúde do Trabalhador pode ser discutido sob vários pontos de vista, e o Ministério da Saúde o define como a ação contínua e sistemática, ao longo do tempo, para detectar, conhecer, pesquisar e analisar fatores tecnológicos, sociais, organizacionais e epidemiológicos relacionados aos processos e ambientes de trabalho, determinando e condicionando os agravos à saúde do trabalhador, tendo como finalidade planejar, executar e avaliar intervenções que eliminem ou controlem seus efeitos deletérios à saúde dos trabalhadores. ${ }^{6}$ O foco em promoção da saúde e a proposta da Vigilância em Saúde vêm se colocando como importantes instrumentos para que a área da Saúde do Trabalhador possa integrar-se e sair do isolamento em que se encontra nas políticas públicas de saúde, podendo promover a visualização de que os agravos relacionados à saúde neste campo, não dizem respeito apenas aos trabalhadores, mas também ao ambiente e à população em geral. ${ }^{7}$

Nessa perspectiva, a notificação dos agravos à saúde do trabalhador ou a falta dessa prática é um fato preocupante para as políticas de saúde desde a década de 80 , muito articulado com insuficientes intervenções das Delegacias Regionais do Trabalho, e dos serviços médicos das empresas. A notificação compulsória de doenças infecto-contagiosas e de agravos relacionados ao trabalho no Sistema de Informação de Agravos de Notificação (SINAN) e no Sistema de Informações em Saúde do Trabalhador (SIST)/RS através do Relatório Individual de Notificação de Agravos (RINA) são instrumentos importantes para que se possa identificar o motivo pelo qual os trabalhadores adoecem ou morrem. Destaca-se também, a identificação dos ramos de atividade econômica e de trabalho, além de fazer intervenções sobre as causas e determinantes e elaborar estratégias de atuação nas áreas de promoção e prevenção, controlando e enfrentando, de forma integrada e eficiente, os problemas de saúde coletiva relacionados com o trabalho. Além destes formulários de notificação dos casos de acidentes e doenças relacionadas ao trabalho, a Comunicação de Acidente de Trabalho (CAT) tem a finalidade de que o acidente seja legalmente reconhecido pelo Instituto Nacional do Seguro Social (INSS) para a obtenção de auxílio e indenizações acidentárias e informações sobre acidentes e doenças do trabalho, direcionando ações em saúde, de forma que os serviços de fiscalização desencadeiem iniciativas que evitem que acidentes e agravos do trabalho se repitam. ${ }^{8}$

Considerando a magnitude e importância deste tema, o objetivo deste estudo é caracterizar dados sociodemográficos e ocupacionais de trabalhadores acometidos por acidentes graves do trabalho no municipio de Santa Cruz do Sul/RS. 


\section{MÉTODOS}

Trata-se de um estudo de campo, documental, retrospectivo, em que os dados foram coletados em formulários de notificações de agravos em saúde do trabalhador (RINA E CAT) no período de 2008 a 2010, disponíveis nos prontuários dos pacientes da Unidade Municipal de Referência em Saúde do Trabalhador (UMREST) do município de Santa Cruz do Sul/RS.

A UMREST é uma unidade de saúde do Sistema Único de Saúde (SUS) especializada em atender os acometidos por acidentes e doenças causadas ou relacionadas ao trabalho, priorizando a assistência de atenção integral à saúde desenvolvendo ações de promoção, prevenção e assistência aos usuários desse serviço. O município de Santa Cruz do Sul localiza-se no Vale do Rio Pardo/RS, distante 155 km da capital Porto Alegre, com uma população estimada em cerca de 120.000 habitantes, segundo dados do censo do IBGE de 2010. Seu principal estímulo econômico vem das plantações de tabaco, que trouxeram para o município inúmeros fabricantes de cigarro e distribuidoras de fumo, apresenta também outros ramos fortes em sua economia como o comércio e serviços, formando uma rede econômica bem estruturada. ${ }^{9}$

Após a coleta, os dados foram digitados no software SPSS 20.0 e analisados estatisticamente, sob forma descritiva, sendo quantificados em frequências absolutas e relativas. Os dados foram organizados por bairro, sendo que os endereços das instituições de trabalho onde ocorreram os acidentes foram georreferenciados por coordenadas UTM (Universal Transversa de Mercator), utilizando a plataforma “Google Earth", este localizou e identificou o bairro do município onde se situavam os estabelecimentos de trabalho, elaborando-se, então, o mapa geográfico.

Este trabalho de mapeamento teve como intenção estimular os serviços envolvidos na assistência à saúde do trabalhador para a importância da atenção integral, fortalecendo a vigilância e a assistência à saúde, favorecendo um cuidado mais aprimorado nos ambientes e processos de trabalho. As técnicas de geoprocessamento são consideradas ferramentas importantes de análise das relações entre o ambiente e eventos relacionados à saúde, devido a sua capacidade de integrar diversas operações que envolvem captura, armazenamento, manipulação, seleção e busca de informação, análise e apresentação de dados, envolvendo planejamento e definições de estratégias no campo da Vigilância em Saúde, especificamente nesse estudo, voltadas às ações de Vigilância em Saúde do Trabalhador. 10,11

O projeto foi desenvolvido de acordo com a Resolução 466/12 que regulamenta a pesquisa com seres humanos e passou pela apreciação do Comitê de Ética em Pesquisa (CEP) 
da Universidade de Santa Cruz do Sul (UNISC) sendo aprovado sob o número de protocolo $2764 / 10$.

\section{RESULTADOS}

Realizou-se levantamento de dados em 1.263 formulários de notificações de pacientes da UMREST. A tabela 1 revela que houve maior frequência de acidentados do trabalho do sexo masculino (78,59\%). Quanto a faixa etária, a maior ocorrência de acidentes de trabalho grave foi entre trabalhadores de 20 a 29 anos, seguido da faixa etária de 30 a 39 anos, o que mostra trabalhadores jovens acometidos por acidentes.

Tabela 1 - Descrição da frequência de acidente grave por sexo e faixa etária.

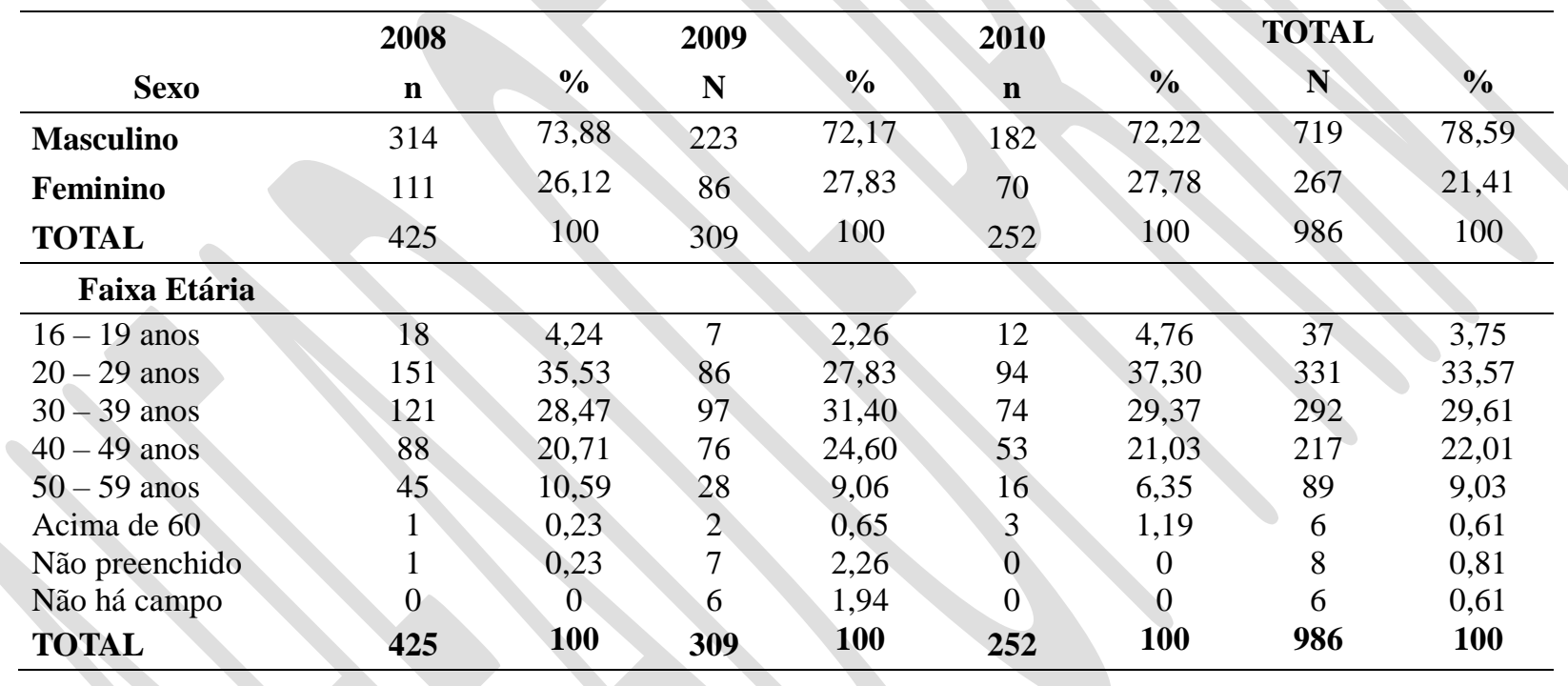

A tabela 2 apresenta o tipo de acidente com maior frequência, que foi o acidente típico, totalizando 657 casos, seguido do acidente de trajeto, com 308 casos. Além disso, houve 16 casos que não continham a informação nas fontes de notificação e ainda cinco casos em que não existia campo para essa informação.

Tabela 2 - Acidente Grave por Tipo de Acidente, Santa Cruz do Sul- RS.

\begin{tabular}{ccccccccc}
\hline & \multicolumn{2}{c}{$\mathbf{2 0 0 8}$} & \multicolumn{2}{c}{$\mathbf{2 0 0 9}$} & \multicolumn{2}{c}{$\mathbf{2 0 1 0}$} & \multicolumn{2}{c}{ TOTAL } \\
Tipo de Acidente & $\mathbf{n}$ & $\mathbf{\%}$ & $\mathbf{n}$ & $\mathbf{\%}$ & $\mathbf{n}$ & $\boldsymbol{\%}$ & $\mathbf{n}$ & $\mathbf{\%}$ \\
\hline Típico & 276 & 64,94 & 207 & 66,99 & 174 & 69,05 & 657 & 66,63 \\
Trajeto & 136 & 32 & 96 & 31,07 & 76 & 30,16 & 308 & 31,24 \\
Não preenchido & 10 & 2,35 & 4 & 1,29 & 2 & 0,79 & 16 & 1,62 \\
Não há campo & 3 & 0,71 & 2 & 0,65 & 0 & 0 & 5 & 0,51 \\
\hline Total & $\mathbf{4 2 5}$ & $\mathbf{1 0 0}$ & $\mathbf{3 0 9}$ & $\mathbf{1 0 0}$ & $\mathbf{2 5 2}$ & $\mathbf{1 0 0}$ & $\mathbf{9 8 6}$ & $\mathbf{1 0 0}$ \\
\hline
\end{tabular}


Referente a função mais acometida, encontram-se os alimentadores de linha de produção com $13,48 \%$, seguido dos auxiliares de processamento de fumo apresentando $8 \%$ e trabalhadores nos serviços de manutenção e conservação de edifícios e logradouros com $7 \%$ dos acidentados (Tabela 3). A função classificada como "Outros" se refere a inúmeras e diferentes ocupações - em torno de 605 ocupações - que apareceram pelo menos uma vez nos formulários pesquisados. A não utilização padronizada das funções descritas no $\mathrm{CBO}$ e a compreensão diferenciada entre as empresas quanto à mesma ocupação, torna obscuro alguns dados. Tal dispersão de ocupações encontradas pode ter ocorrido devido às muitas formas de nominar uma mesma função/ocupação.

Tabela 3 - Acidente Grave por Função, Santa Cruz do Sul-RS.

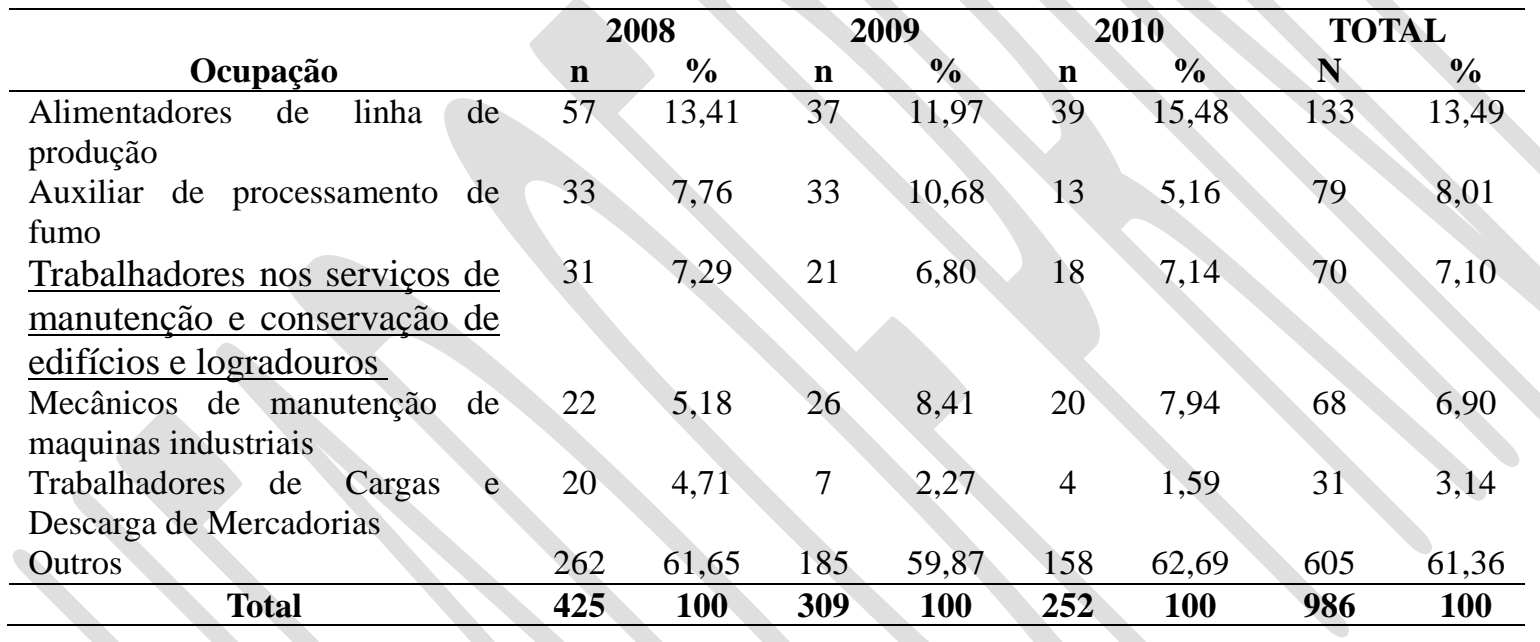

Quanto à localização geográfica das instituições onde ocorreram os acidentes, das 986 ocorrências de acidentes graves de trabalho, 463 ocorreram em bairros da região central de Santa Cruz do Sul, perfazendo 47,2\% dos casos, seguido pelo bairro denominado Distrito Industrial, localizado na região sul do município, com $27,1 \%$ dos casos. O restante das ocorrências, representada por um número expressivamente menor, encontra-se distribuído nos demais bairros do município. A fim de visualizar a distribuição dos casos encontrados, estes foram inseridos no mapa do município de Santa Cruz do Sul, distribuídos por bairros com maior número de casos (Figura 1). 
Figura 1 - Mapa de geolocalização de casos de acidentes de trabalho grave no município de Santa Cruz do Sul-RS.

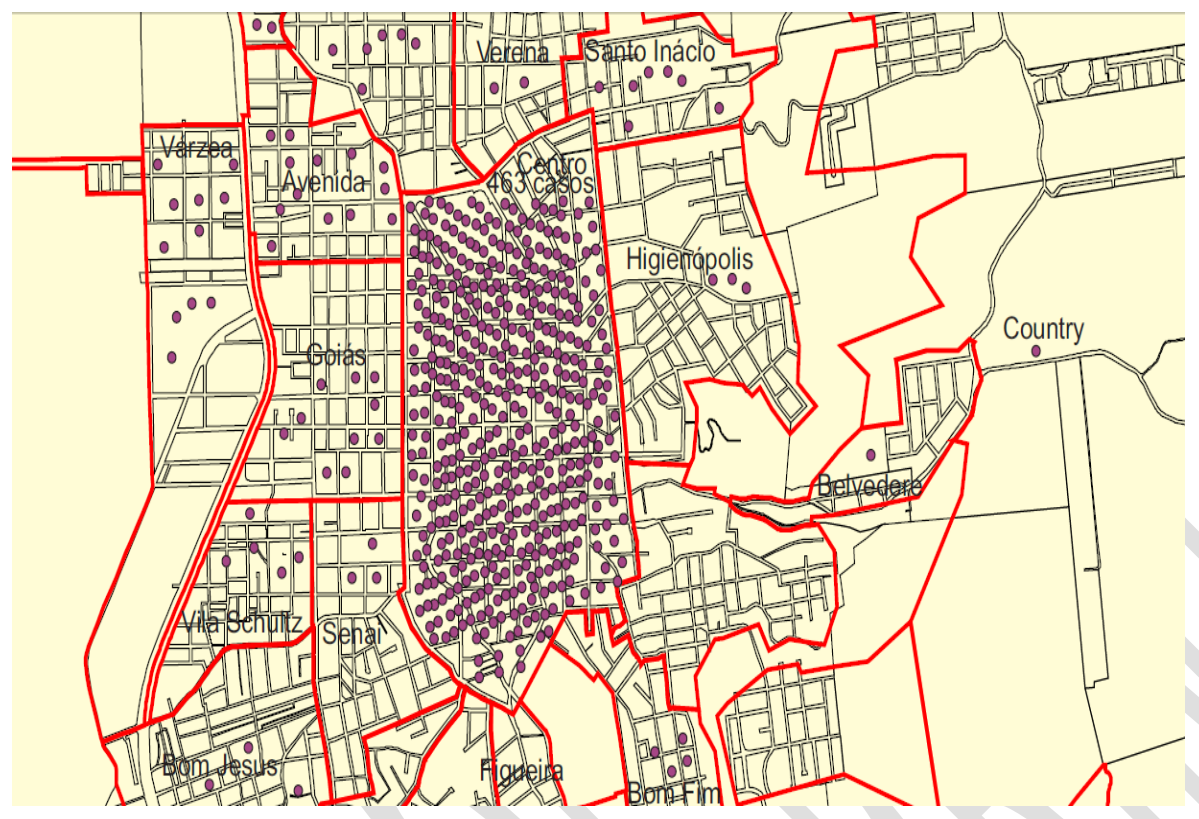

O estudo não efetuou análise estatística no que se refere à relação entre o tipo de acidente - típico e de trajeto - e o bairro de localização geográfica das instituições onde os acidentes ocorreram.

\section{DISCUSSÃO}

O estudo revelou que houve uma maior frequência de acidentes de trabalho grave em indivíduos do sexo masculino, corroborando com estudos realizados no Rio Grande do Norte que observou 91,8\%, no estado do Paraná com 88,4\% e em Campinas com 92,5\%. ${ }^{12-14}$

Quanto a faixa etária, a maior ocorrência de acidentes de trabalho grave foi entre trabalhadores de 20 a 29 anos, o que, de acordo com a literatura, pode ser atribuída à elevada participação deste grupo etário mais jovem na força de trabalho e, em especial, naquelas atividades com maior grau de risco. ${ }^{15}$ Estudos realizados sobre acidentes de trabalho no Brasil, revelaram maior prevalência dos casos na menor faixa etária produtiva, logo após os 18 anos, trabalhadores com idade entre 26 e 30 anos $(17,1 \%)^{13}$ e entre $18-29(47,5 \%) .{ }^{4,14}$

$\mathrm{O}$ acidente com maior prevalência no estudo foi o acidente típico, que é aquele que ocorre durante o desempenho laboral, semelhante ao estudo realizado no Estado do Paraná que revelou predominância dos acidentes típicos $(73,9 \%)$ e ao estudo realizado no Rio Grande do Norte que observou predominância de acidentes típicos (76,3\%). ${ }^{12,13,16}$ 
A função observada com maior frequência foi dos alimentadores de linha de produção, semelhante aos dados do Ministério da Previdência Social, revelando que no ano de 2006, os trabalhadores de funções industriais foram os mais acometidos por acidentes típicos, com 13,3\% do total. ${ }^{17}$ Outros estudos também constataram que a maior parte dos acidentes atinge homens jovens e produtivos, que trabalham nas indústrias, na construção civil, no setor de transportes e na agricultura, locais onde mais ocorrem acidentes graves. ${ }^{18,19}$

Os bairros localizados na região central e sul do município apresentaram maior ocorrência de acidentes de trabalho graves, possivelmente, devido a maior concentração geográfica, respectivamente, de instituições dos setores econômicos de comércio (3.277 estabelecimentos), de prestação de serviços (2.793 estabelecimentos) e indústrias, principalmente do ramo fumageiro (17 estabelecimentos). De acordo com esse achado, no estudo realizado no estado do Rio Grande do Norte, os acidentes graves, em sua maioria, foram identificados em municípios mais desenvolvidos economicamente, não somente no segmento industrial, mas em segmentos como o da construção civil e da agricultura, esta ultima pela mecanização. ${ }^{20}$

Por fim, conclui-se que o estudo mostrou a ocorrência de 986 casos de acidentes graves do trabalho no município de Santa Cruz do Sul/RS no período de 2008 a 2010, sendo que no ano de 2008 houve maior incidência de casos notificados nos sistemas de informação da área de Saúde do Trabalhador. Os acidentes acometeram, com maior frequência, trabalhadores do sexo masculino, na faixa etária de 20 a 39 anos, alocados em instituições localizadas em bairros de predominância dos segmentos de comércio e serviços da região central do município.

Os resultados deste estudo evidenciam a situação epidemiológica do trabalhador acometido por acidente de trabalho grave no município. Ressalta-se, dessa maneira, a importância de enfatizar a assistência à saúde do trabalhador, formulando estratégias de prevenção, proteção e orientação tanto aos trabalhadores quanto às empresas, considerando a diversidade econômica e geográfica. Esses propósitos vêm ao encontro do preconizado pela Política Nacional de Saúde do Trabalhador do Ministério da Saúde, que tem como objetivo reduzir a ocorrência dos acidentes de trabalho e das doenças ocupacionais, propondo ações de promoção, reabilitação e vigilância na área da saúde, identificando a relação entre ambiente, organização, condições de trabalho e as implicações desse imbricamento de relações sobre a saúde do trabalhador. 
Como limitação do estudo, pode ser apontado o uso dos dados produzidos pelos sistemas de vigilância e informação, devido a possibilidade de subnotificação de casos, o que dificulta um diagnóstico situacional da Vigilância em Saúde do Trabalhador.

\section{REFERÊNCIAS}

1. Junqueira AGW, Franz SM. Sentido do trabalho: percepção dos professores universitários de uma Instituição de Ensino Superior. Estudo \& Debate, Lajeado 2012; 19(1): 51-75.

2. Ministério da Previdência Social (BR). Proteção para o Trabalhador e sua família - MPS. Saúde e Segurança Ocupacional. Brasília 2012.

3. Ministério da Saúde (BR). Portaria n 3.252, de 22 de dezembro de 2009. Aprova as diretrizes para execução e financiamento das ações de Vigilância em Saúde pela União, Estados, Distrito Federal e Municípios e dá outras providências. Diário Oficial da União, Brasília, n. 245, Seção 1, p. 65-69, 23 de dezembro de 2009.

4. Almeida PCA, Barbosa-Branco A. Acidentes de trabalho no Brasil: prevalência, duração e despesa previdenciária dos auxílios-doença. Rev Bras Saúde Ocup 2011; 36(124):195-207. DOI: 10.1590/S0303-76572011000200003.

5. Iwamoto HH, Camargo FC, Tavares LC, et al. Acidentes de trabalho fatais e a qualidade das informações de seus registros em Uberaba, em Minas Gerais e no Brasil, 1997 a 2006. Rev Bras Saúde Ocup 2011; 36(124): 208-215. DOI: 10.1590/S0303-76572011000200004.

6. Ministério da Saúde (BR). Portaria $n^{\circ} 3.120$, de $1^{\circ}$ de julho de 1998. Aprova a Instrução Normativa de Vigilância em Saúde do Trabalhador no SUS. Diário Oficial da União, Brasília, n. 124, Seção 1, p. 36-38, 2 de julho de 1998.

7. Alves RB. Vigilância em saúde do trabalhador e promoção da saúde: aproximações possíveis e desafios. Cad Saúde Pública 2003; 19(1): 319-322. DOI:10.1590/S0102311 X2003000100036. 
8. Cordeiro R, Sakate M, Clemente APG, et al. Subnotificação de acidentes do trabalho não fatais em Botucatu, SP, 2002. Rev Saúde Pública 2005; 39(2): 254-260. DOI: 10.1590/S003489102005000200017.

9. Secretaria Municipal da Fazenda - Prefeitura Municipal de Santa Cruz do Sul; Economia Indústria, Comércio, Serviços e Autônomos, Santa Cruz do Sul, 2011.

10. Barcellos C, Ramalho WM, Gracie R, et al. Georreferenciamento de dados de saúde na escala submunicipal: algumas experiências no Brasil. Epidemiol Serv Saúde 2008; 17(1):5970. DOI: $10.5123 /$ S1679-49742008000100006

11. Muller EPL, Cubas MR, Bastos LC. Georreferenciamento como instrumento de gestão em unidade de saúde da família. Rev Bras Enferm 2010; 63(6): 978-982. DOI: 10.1590/S003471672010000600017.

12. Cavalcante CAA, Medeiros SM, Mata MS, et al. Acidentes de trabalho grave no Rio Grande do Norte: estudo transversal. Online braz j nurs [internet] 2015 [citado 2016 set 16]; 14:543-55. Disponível em: http://www.objnursing.uff.br/index.php/nursing/article/view/5221

13. Scussiato LA, Sarquis, LMM, Kirchhofet, ALC, et al. Perfil epidemiológico dos acidentes de trabalho graves no Estado do Paraná, Brasil, 2007 a 2010. Epidemiol Serv Saúde 2013; 22(4): 621-630. DOI: 10.5123/S1679-49742013000400008

14. Farias SH, Lucca SR. Perfil dos trabalhadores vítimas de acidente de trabalho grave usuários de prótese do programa de readaptação profissional. Ver Baiana Saúde Pública 2013; 37(3): 725-738.

15. Faria MP, Silva AM. Análise dos acidentes ocorridos durante parte do ano de 1983 na Grande Belo Horizonte (MG). Rev Bras Saúde Ocupacional 1986; 12(53): 26-32.

16. Sêcco IAO, Robazzi MLCC, Shimizu DS, et al. Acidentes de trabalho típicos envolvendo trabalhadores de hospital Universitário da região sul do Brasil: Epidemiologia e prevenção. Rev Latino-am Enfermagem 2008; 16(5). DOI: 10.1590/S0104-11692008000500005 
17. Ministério Da Previdência Social (BR). Estatísticas. Anuário Estatístico da Previdência Social 2006. Seção IV - Acidentes do Trabalho. Disponível em: http://www1.previdencia.gov.br/aeps2006/15_01_03_01.asp

18. Santos SS, Costa NA, Mascarenhas MDM. Caracterização das exposições ocupacionais a material biológico entre trabalhadores de hospitais no Município de Teresina, Estado do Piauí, Brasil, 2007 a 2011. Epidemiol Serv Saude 2013; 22(1): 165-70.

19. Gomez MG, Lopez RCC. Desigualdades interterritoriales em lacompensacion de las enfermedades profesionales em España de 1990 a 2007. Gac Sanit 2009; 23(5): 373-9.

20. Cavalcante CAA, Santos RS, Cavalcante EFO, et al. Perfil dos agravos relacionados ao trabalho notificados no Rio Grande do Norte, 2007 a 2009. Epidemiol Serv Saúde [Internet] 2

0

1

4 